\title{
Anther culture of Lupinus angustifolius: callus formation and the development of multicellular and embryo-like structures
}

\author{
Kamila Kozak • Renata Galek • \\ Mohammad Tahir Waheed • \\ Ewa Sawicka-Sienkiewicz
}

Received: 1 June 2011/Accepted: 18 November 2011/Published online: 30 November 2011

(C) The Author(s) 2011. This article is published with open access at Springerlink.com

\begin{abstract}
Androgenesis is an important technique to generate double haploid plants. Anther and microspore cultures are the methods to induce haploid embryogenesis. For culture initiation, it is necessary to select anthers with the appropriate developmental stage of microspores. For lupins, limited reports about the establishment of initial cultures for androgenesis are available. In this study, different parameters of anther culture of three genotypes of Lupinus angustifolius were investigated. For all genotypes, a considerable correlation was observed between the buds and the anthers, depending on their location in the inflorescences. Buds from the central segment of inflorescences had yellowish green anthers that contained the maximum number of microspores at uninucleate stage. Cytological investigation shows that the anthers containing these microspores were the most responsive to induction. Two types of developmental pathways were observed for microspores. In case of cold pre-treated and untreated inflorescences, microspores developed into multicellular and embryo-like structures, respectively. Effects of different factors showed significant differences among: genotypes, pre-treatment, growth regulators (GRs) and genotypes $\times$ GRs interaction. Among three genotypes, Emir showed the highest number of multicellular and embryo-like structures
\end{abstract}

\footnotetext{
K. Kozak $(\bowtie) \cdot$ R. Galek · E. Sawicka-Sienkiewicz Department of Genetics, Plant Breeding and Seed Production, Wroclaw University of Environmental and Life Sciences, Pl. Grunwaldzki 24 A, 50-363 Wrocław, Poland e-mail:kamikoz@o2.pl

\section{T. Waheed}

Department of Applied Plant Sciences and Plant Biotechnology (DAPP), University of Natural Resources and Applied Life Sciences (BOKU), Gregor-Mendel-Strasse 33, 1180 Vienna, Austria
}

on MS medium $+2.0 \mathrm{mg} / \mathrm{l} 2,4 \mathrm{D}+0.5 \mathrm{mg} / \mathrm{l}$ Kinetin (Kin). For all genotypes, anthers produced calli on MS medium containing $2.0 \mathrm{mg} / \mathrm{l} 2,4 \mathrm{D}+0.5 \mathrm{mg} / \mathrm{l} \mathrm{Kin}$. These calli continued their growth on regeneration medium $(\mathrm{MS}+2.0 \mathrm{mg} / \mathrm{l}$ BA $+0.5 \mathrm{mg} / \mathrm{l}$ NAA) and produced roots. Taken together, these results provide a good basis for further research towards the development of haploid plants for L. angustifolius.

Keywords Androgenesis - Anther culture - Legumes · Lupinus angustifolius · Double haploids · Embryo-like structures

\section{Introduction}

Lupin (family Fabaceae) is cultivated for three main reasons: as a ruminant feed, as a green manure to improve soil structure, and for human nutrition because of their high protein and oil contents, which, depending on the variety, varies from 30 to $50 \%$ and 5 to $10 \%$, respectively (Huyghe 1997; Faluyi et al. 2000). Moreover, lupin is a promising candidate to be used for phytoremediation. In Europe, three species of lupin are commonly cultivated: Lupinus angustifolius, L. albus and L. luteus. Nowadays, the European agricultural focus is upon Lupinus angustifolius, the narrow-leafed lupin, due to its advantages compared to other lupin species: the highest resistance to anthracnose (fungal disease caused by Colletotrichum gloeosporioides), high yield and short vegetation period.

It is very time consuming to produce new cultivars by using conventional breeding methods such as hybridization or induced mutation. In contrast, androgenesis appears to be a more suitable technique in breeding processes. In this phenomenon, under certain conditions, immature pollen 
grains (microspores) can be shifted from the gametophytic to the sporophytic pathway, resulting in the formation of haploid embryos, which then convert to haploid plants (Ferrie and Caswell 2011). To induce androgenesis, anther and microspore cultures are common techniques for obtaining double haploid (DH) plants. The application of double-haploid techniques allow homozygosity to be achieved in a single laboratory-based generation (Croser et al. 2006), hence accelerating the improvement of cultivars and enabling a timely response to market requirements.

Legume species are considered recalcitrant to DH production (Ferrie and Caswell 2011). Successful induction of androgenesis with subsequent regeneration of complete haploid plants is limited to the following legume species: Pisum sativum, Lathyrus sativus, Medicago truncatula, M. sativa and M. arborea (Zagorska et al. 1997; Lanas et al. 2006; Ochatt et al. 2009), Glycine max (Zhao et al. 1998; Moraes et al. 2004), Cajanus cajan (Kaur and Bhalla 1998), Cicer arietinum (Grewal et al. 2009) and for only one legume tree, Swainsona formosa (Sudhersan et al. 2008). For lupin, some existing reports about the induction of haploid embryogenesis show that either early stages of nuclear division, multicellular structures (MCS) or embryo-like structures (ELS) were observed (Sator et al. 1983; Sator 1985; Ormerod and Caligari 1994; Bayliss et al. 2004; Skrzypek et al. 2008). However, none of the protocols was successful in obtaining haploid or DH plants.

An important parameter for successful androgenesis is the selection of buds and anthers with the proper microspore developmental stage (Constantin 1981). In most plant species, microspores at the uninucleate stage are the most responsive to induction. It has been shown that a significant relationship exists between floral bud size and corresponding microspore developmental stages for soybean cultivars (Lauxen et al. 2003). Later, Skrzypek et al. (2008) considered some parameters to study such correlation for Lupinus albus, L. angustifolius and L. luteus.

The aim of the current study was to determine the optimum conditions for the initiation of anther culture for three genotypes of Lupinus angustifolius. Here, we present a comprehensive study correlating the localization and size of buds with size and colour of anthers containing the optimal developmental stage of microspores for the induction of androgenesis. We report callus production and root formation from anthers of all three genotypes of L. angustifolius. Moreover, we have studied the effect of different factors such as cold pre-treatment and medium composition on the induction of embryogenesis by cytological investigation. In case of cold pre-treated and untreated inflorescences, two distinct developmental pathways are reported for microspores, leading to the formation of MCS and ELS, respectively.

\section{Materials and methods}

Plant material and growth conditions

Two commercial cultivars, Emir and Graf (released by Hodowla Roślin Smolice Sp. z o. o Grupa IHAR, Smolice, Poland) and the breeding line LAE-I of Lupinus angustifolius $(2 \mathrm{n}=40)$ were used in this study. To obtain anthers, donor plants were grown under natural conditions, at the experimental field of Department of Genetics, Plant Breeding and Seed Production in Wrocław (Poland). The seeds were sown on April 2nd 2007, and the young inflorescences of field grown plants were harvested between 7.30 a.m. and 9.30 a.m. on May 31st 2007 (10-year average temperature in April and May $9.3^{\circ} \mathrm{C}$ and $14.6^{\circ} \mathrm{C}$, respectively; 10-year average rain fall in April and May 33.8 and $54.1 \mathrm{~mm}$, respectively). The soil was provided with fertilizer containing nitrogen $(32 \mathrm{~kg} / \mathrm{ha})$, potassium $(96 \mathrm{~kg} / \mathrm{ha})$ and phosphorus $(96 \mathrm{~kg} / \mathrm{ha})$. For all genotypes, the young inflorescences were collected from the main stem of the plants. Buds contained in the inflorescences were closed at the time of collection. These inflorescences were transported from the experimental field to the laboratory at $4^{\circ} \mathrm{C}(20 \mathrm{~min})$ and their lengths were measured. The inflorescences were either subjected to cold pre-treatment for 4 days at $4^{\circ} \mathrm{C}$ in the dark or used directly for culture initiation after transportation. Ten inflorescences from each genotype were selected and each inflorescence was separated into three parts: segment I (the apical portion), segment II (the middle portion) and segment III (the basal portion). Depending on the developmental stages of microspores, the anthers from segment II were selected for culture.

\section{Culture conditions}

Freshly cut or cold pre-treated inflorescences were surface sterilised with $70 \%(\mathrm{v} / \mathrm{v})$ ethanol for $2 \mathrm{~min}$ and $6 \%(\mathrm{w} / \mathrm{v})$ calcium hypochlorite solution for $10 \mathrm{~min}$, followed by three washings with sterile distilled water (10 min each). The induction medium consisted of MS (Murashige and Skoog 1962) medium containing mineral salts, vitamins and 3\% (w/v) sucrose. This medium was supplemented with $2.0 \mathrm{mg} / \mathrm{l}$ 2,4-dichlorophenoxyacetic acid (2,4-D, control medium) combined with one of the following growth regulators (GRs): $0.5 \mathrm{mg} / \mathrm{l}$ 6-benzyladenine (BA), $0.5 \mathrm{mg} / \mathrm{l} \alpha$-naphthaleneacetic acid (NAA) or $0.5 \mathrm{mg} / \mathrm{l}$ kinetin (Kin). The $\mathrm{pH}$ was adjusted to 6.0 , before adding $0.8 \%$ agar. Media were autoclaved at $121^{\circ} \mathrm{C}$ for $20 \mathrm{~min}$. The anthers were placed in Petri dishes $(\varnothing 3 \mathrm{~cm})$ containing the desired induction medium and incubated in the dark for 28 days at $24^{\circ} \mathrm{C}$. After the initial culture of 28 days, the anthers were placed on regeneration medium 
(MS medium containing $2.0 \mathrm{mg} / \mathrm{l} \mathrm{BA}+0.5 \mathrm{mg} / \mathrm{l} \mathrm{NAA}$ ) and shifted to climatic chamber at $24^{\circ} \mathrm{C}, 16 / 8 \mathrm{~h}$ (light/dark) and light intensity of $100 \mu \mathrm{m} / \mathrm{m}^{2} / \mathrm{s}$. During this period, the anthers were checked daily.

\section{Microscopic analysis}

Anthers collected from buds of all three segments were measured and their colour was determined. To study the viability and the developmental stage of the microspores, these anthers were fixed in Carnoy's solution (3:1 mixture of absolute ethanol and acetic acid) and stored in 70\% (v/v) ethanol until investigation. Squashed preparations were used to observe viability and developmental stages of microspores. Hundred pollen grains per anther were counted in randomly chosen fields of vision using $100 \times$ magnification. For squashing, either standard acetocarmine procedure was used and visualized under light microscope (Nikon Eclipse 200), or with one drop of DAPI (4,6 diamidino-2-phenylindol) and observed by using a fluorescent microscope (Nikon E 400). Based on morphological criteria such as cell size and shape (Erdtman 1952) and position of the nucleus, different stages of microspore development were established and counted. Microspores that were not stainable through acetocarmine staining were considered as non-viable. During the whole induction period (28 days), squashed preparations of randomly chosen anthers were used to study the development of microspores, using both above mentioned microscopic procedures. The cultured anthers were studied at days 4th, 7th, 14th, 21st and 28th after the start of the culture.

\section{Statistics}

The experiments were devised as complete randomized design. Each treatment consisted of 10 replicates and one petri dish containing 50 anthers of one genotype on one type of medium was regarded as one replicate. MCS and ELS were counted per anther after 28 days of culture initiation. The data obtained were statistically analyzed by analysis of variance (STATISTICA, version 8.0). Mean values were evaluated at $P=0.05$ level of significance and compared using Duncan's test.

\section{Results}

Correlation of bud size, anthers and developmental stage of microspores

For all genotypes, the characteristics of inflorescences were identical. The average lengths of inflorescences were $3-5 \mathrm{~cm}$. There was no difference in the bud sizes among three genotypes. Segments I, II and III (apical, central and basal segments, respectively) of inflorescences contained buds of different sizes (Fig. 1). The anthers of the segment I were the smallest and green in colour while the anthers collected from the segments II and III were yellowish green and bright yellow, respectively.

Cytological study shows five different developmental stages of the pollen grains, depending on their localization within the anthers of the three segments. Results show that developmental stages of the pollen grains were not synchronized within these anthers. The youngest anthers (segment I) contained tetrads to mid-uninucleate stages (Fig. 1a, c): mainly early-uninucleate microspores (55\%), freshly released from callose wall. These microspores exhibited a centrally located nucleus containing non-condensed components (Fig. 1b). The microspores from the anthers of segment II displayed tetrads to mature developmental stages (Fig. 1a, e). In the anthers from segment II, late-uninucleate stage of microspores was predominant (51\%) having a condensed nucleus that shifted to the pollen wall (Fig. 1d). Microspores obtained from anthers of segment III entirely contained advanced developmental stages, representing 64\% mature pollen grains (Fig. 1e). Based on the results, for each genotype, anthers from segment II were used for culture. The data shown in Fig. 1 is the average for three genotypes of Lupinus angustifolius. In Fig. 2, the cytological study of the developmental stages of microspores is depicted individually for Emir, Graf and LAE-1.

Viability of microspores and pollen prior to the culture

Microspores viability from anthers obtained from the three segments of inflorescences was studied. For genotypes Emir and Graf, the maximum number of viable pollen (98 and $97 \%$, respectively) was obtained from anthers of segment II (Fig. 2). For LAE-1, pollen from anthers of the segment III showed the highest viability (97\%, Fig. 2). The lowest proportion of non-viable pollen (3\%) was observed from anthers of segment II (Fig. 1). The data given above is for untreated inflorescences. In case of cold pre-treated inflorescences, the viability of microspores was identical (data not shown).

\section{Development of microspores in culture}

To study androgenesis, microspores were dissected from the cold pre-treated and untreated cultured anthers. In our cytological study we observed no response from microspores at the tetrad and early-uninucleate stages of development for all genotypes. These microspores remained stainable for several days. In some microspores, nuclei started to condense and moved to the pollen wall 


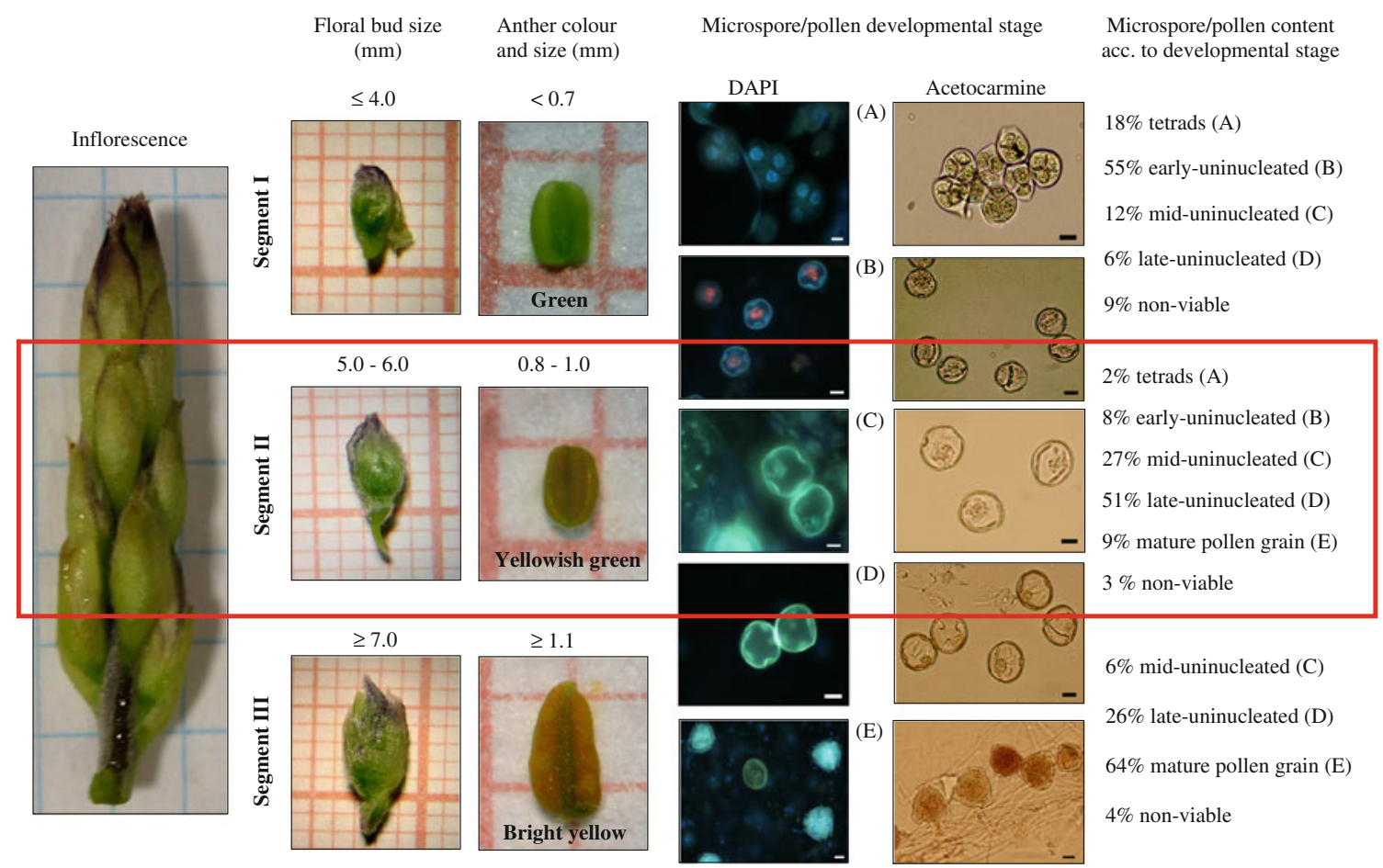

Fig. 1 Correlation of bud size, anther size and colour, and the developmental stages of microspores and pollen. Data are shown as average for three genotypes of $L$. angustifolius, from three segments of inflorescences. bar $=10 \mu \mathrm{m}$

Fig. 2 Distribution of developmental stages of microspores and pollen grains along the young inflorescences of Lupinus angustifolius varieties 'Emir', 'Graf' and breeding line 'LAE-1'. Inflorescences of 3-5 cm length were divided into 3 segments: the apical segment $-I$; the middle segment-II, the basal segment-III

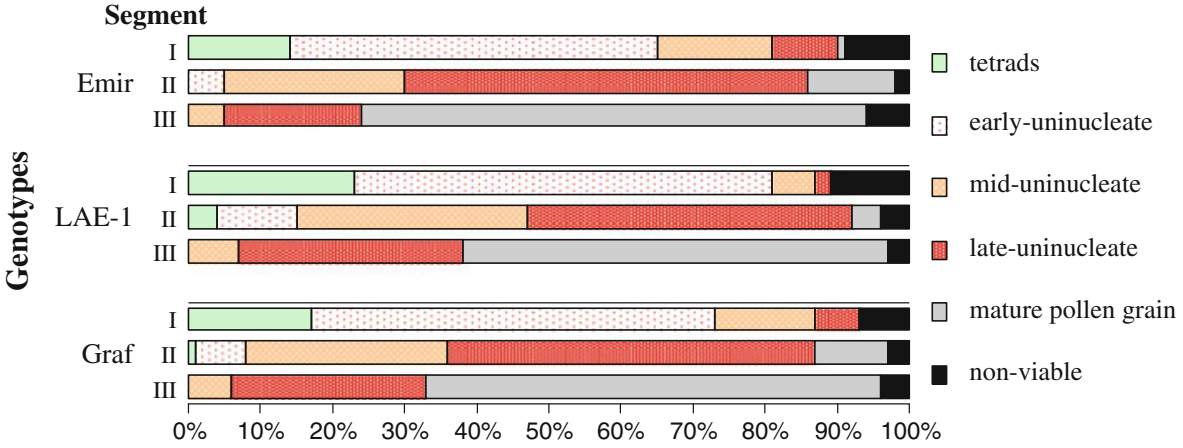

(sporodermis). However, most of these microspores degenerated between day 7 and 14. Most cells showing signs of androgenic induction were found in anthers from the middle segment of the inflorescences, where microspores at midand late-uninucleate stages were in the majority. For all genotypes, cold pre-treated and untreated inflorescences showed two distinct pathways of microspore development (Fig. 3). In both treated and untreated inflorescence, observation (day 4) showed that nuclei of microspores became condensed and moved to the exine sporoderm (Fig. 3B). After this period, the first difference in response was observed between the microspores of treated and untreated inflorescences. In case of untreated inflorescences, between days 4 and 7 , the nucleus of the microspore divided symmetrically into two vegetative nuclei (Fig. 3C). Between days 7 and 21, the vegetative nucleus continued dividing and the diameter of the microspores increased to $20 \mu \mathrm{m}$ (Fig. 3D, E). At the end of the culture period (day 28), due to the continuous division of nuclei, microspores developed into multicellular structures with a diameter of $\sim 50 \mu \mathrm{m}$ (Fig. 3F). In case of treated inflorescences, between day 0 and 4 , microspores started growing in size (from $\sim 12$ to $17 \mu \mathrm{m})$ due to the accumulation of granules (Fig. 3b). Between days 4 and 7 , the accumulation of granules increased and nuclear division was not visible (Fig. 3c). By day 7, the microspore diameter had increased to $20 \mu \mathrm{m}$. Between days 14 and 21, the whole cytoplasm was filled by granules (Fig. 3d) and finally at the end of induction period, a compact oval shaped embryo-like structure was formed, having diameter of $70 \mu \mathrm{m}$ (Fig. 3e). 


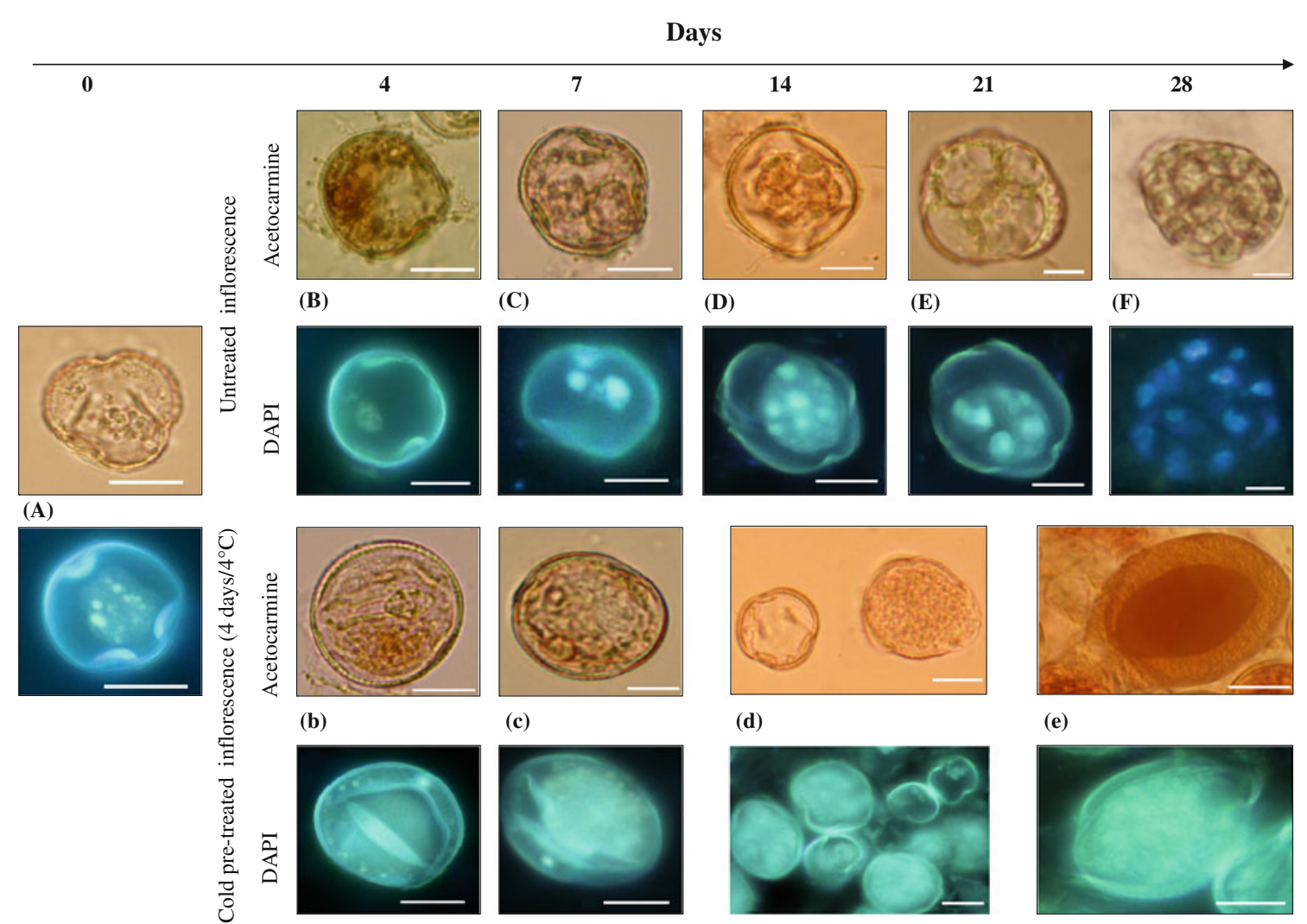

Fig. 3 Comparison of developmental pathways of microspores from anthers of cold pre-treated and untreated inflorescences, leading to the formation of multicellular and embryo-like structures. The

Formation of callus from anthers

For all genotypes, callus formation was only observed from few anthers on the MS medium supplemented with $2.0 \mathrm{mg} /$ 1 2,4-D and $0.5 \mathrm{mg} / \mathrm{l} \mathrm{Kin}$. The first callus was observed after 3 weeks from the start of the culture. These calli were white in colour and either compact or friable (Fig. 4a, b) that further developed into white structures resembling embryos (Fig. 4a). After 1 week on regeneration medium (MS medium supplemented with $2.0 \mathrm{mg} / \mathrm{l} \mathrm{BA}+0.5 \mathrm{mg} / \mathrm{l}$ NAA), the calli became green (Fig. 4c) and after 2 weeks, the calli formed roots (Fig. 4d, e). However, at the end of 1 month on regeneration medium, all the calli turned brown and died (Fig. 4f).

\section{Effects of different factors on androgenesis}

The influence of three factors (genotype, pre-treatment and GR combinations) on androgenesis was studied. A total of 2,205 anthers were screened for the development of MCS and ELS. Effects of these factors are presented in Table 1. The analysis of data detected statistically significant differences among: genotypes, pre-treatment, GRs (Table 1) and interaction genotypes $\times$ GRs (Fig. 5). microspores presented here were cultured on MS medium containing $2.0 \mathrm{mg} / \mathrm{l}$ 2,4-D + $0.5 \mathrm{mg} / \mathrm{l} \mathrm{Kin.} \mathrm{Bar} \mathrm{A,} \mathrm{B,} \mathrm{C,} \mathrm{D,} \mathrm{E,} \mathrm{b,} \mathrm{c} \mathrm{=} 10 \mu \mathrm{m}$; $\mathbf{d}=15 \mu \mathrm{m} ; \mathbf{F}, \mathbf{e}=20 \mu \mathrm{m}$

Among the three examined genotypes, the mean of MCS and ELS obtained per anther was greater for cv. Emir (1.05) and lowest for breeding line LAE-1 (0.61) (Table 1). MS medium containing $2.0 \mathrm{mg} / \mathrm{l}$ of 2,4-D $+0.5 \mathrm{mg} / \mathrm{l} \mathrm{Kin}$ showed the best induction among all tested media, for all genotypes. Cold pre-treatment of inflorescences promoted the induction in all the examined genotypes, forming approximately one MCS and ELS per anther, compared to 0.7 in case of untreated inflorescences (Table 1). The interaction genotype $\times$ GRs showed that for all genotypes, medium containing $2.0 \mathrm{mg} / \mathrm{l}$ of $2,4 \mathrm{D}+0.5 \mathrm{mg} / \mathrm{l} \mathrm{Kin}$ produced the maximum number of MCS and ELS. Among the three genotypes, cv. Emir exhibited the highest number of these structures (1.8) per anther on the above mentioned medium (Fig. 5).

\section{Discussion}

In the present study, we determine the optimal size of the buds and the anthers with the appropriate developmental stage of microspores for the induction of androgenesis. A considerable correlation is found between bud size, anthers and the developmental stages of microspores. We 

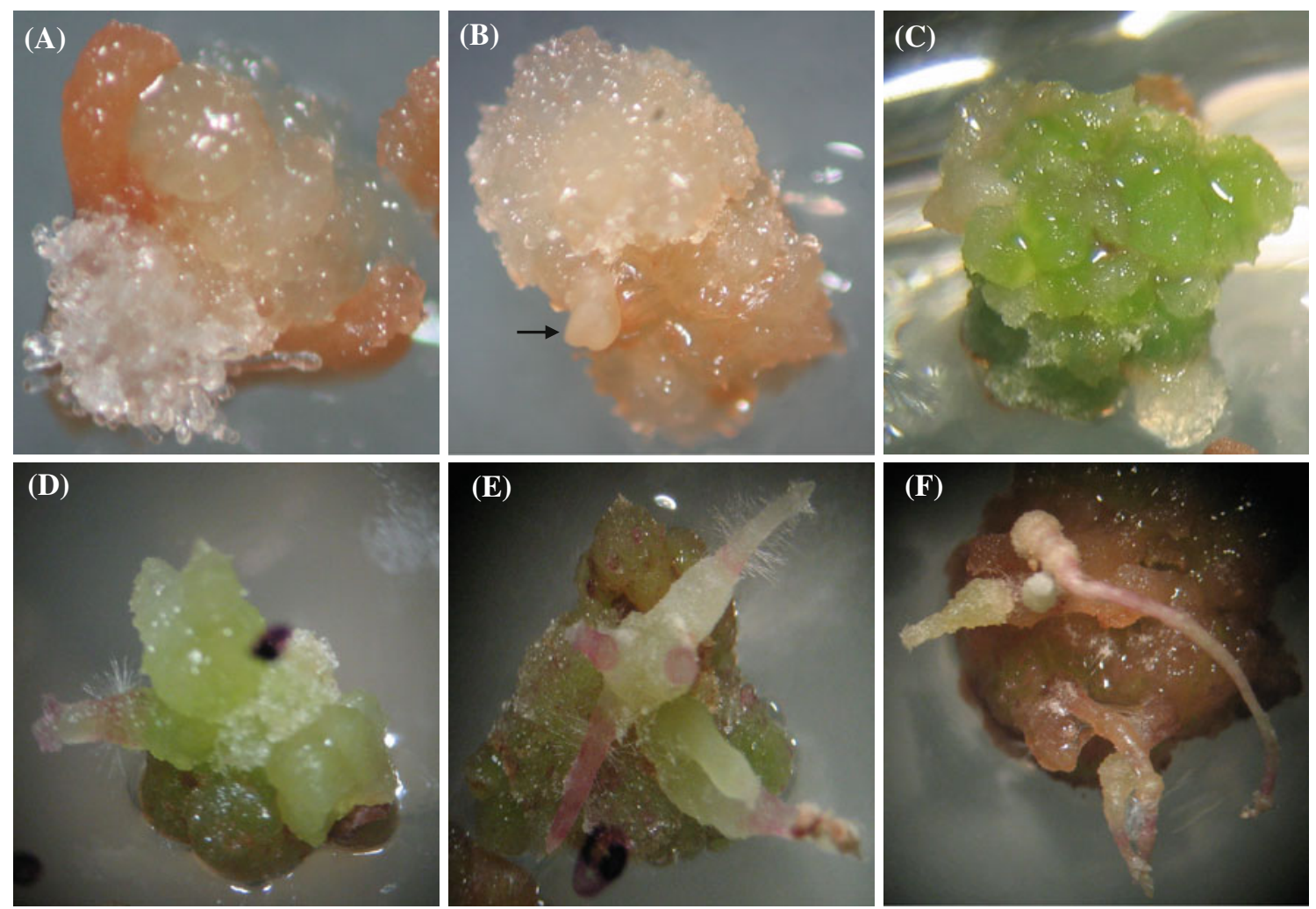

Fig. 4 Callus formation from anthers of three genotypes of $L$. angustifolius. A and $\mathbf{B}$ White callus produced on MS medium containing $2.0 \mathrm{mg} / \mathrm{l}$ 2,4-D and $0.5 \mathrm{mg} / \mathrm{l} \mathrm{Kin}$, after 3 weeks of initial culture (starting from day 0), arrow shows the embryo-like structure;
C green calli after 1 week on regeneration medium (MS medium supplemented with $2.0 \mathrm{mg} / \mathrm{l} \mathrm{BA}+0.5 \mathrm{mg} / \mathrm{l} \mathrm{NAA}$ ); $\mathbf{D}$ and $\mathbf{E}$ root formation on regeneration medium after 2 weeks on regeneration medium; $\mathbf{F}$ brown callus after 4 weeks on regeneration medium
Table 1 The effect of genotype, cold pre-treatment and composition of growth regulators in the media on the number of induced multicellular structures and embryo-like structures found 4 weeks after the start of the culture

* MCS and ELS were counted at the end of the induction period (day 28), $a, b, c$ mean values followed by the same letter are not significantly different at $P=0.05$ according to Duncan's test

\begin{tabular}{lccc}
\hline Factors & $\begin{array}{l}\text { Number of } \\
\text { anthers cultured }\end{array}$ & $\begin{array}{l}\text { Number of anthers } \\
\text { observed under } \\
\text { microscope }\end{array}$ & $\begin{array}{l}\text { Number of MCS } \\
\text { and ELS counted } \\
\text { per anther* }\end{array}$ \\
\hline $\begin{array}{l}\text { Genotypes } \\
\text { Emir }\end{array}$ & 3,948 & 775 & $1.05 \mathrm{a}$ \\
Graf & 3,623 & 707 & $0.87 \mathrm{~b}$ \\
LAE-1 & 3,536 & 723 & $0.61 \mathrm{c}$ \\
Pre-treatment & & & $0.70 \mathrm{~b}$ \\
0 days $/ 4^{\circ} \mathrm{C}$ & 5,504 & 1,067 & $0.99 \mathrm{a}$ \\
4 days $/ 4^{\circ} \mathrm{C}$ & 5,603 & 1,112 & $1.31 \mathrm{a}$ \\
Composition of growth regulators $(\mathrm{mg} / \mathrm{l})$ & & $0.86 \mathrm{~b}$ \\
$2.02,4-\mathrm{D}+0.5$ Kin & 2,732 & 549 & $0.68 \mathrm{c}$ \\
$2.02,4-\mathrm{D}+0.5$ BA & 2,741 & 543 & $0.58 \mathrm{c}$ \\
$2.02,4-\mathrm{D}+0.5$ NAA & 2,815 & 541 & 546 \\
$2.02,4-\mathrm{D}$ & 2,819 & & \\
\hline
\end{tabular}

reveal that for all genotypes of $L$. angustifolius, microspores located in the anthers of the middle part (segment II) of inflorescences contain the best developmental stage of microspores for further in vitro culturing. A study of different factors illustrates that cold pre-treatment and medium containing $2.0 \mathrm{mg} / \mathrm{l}$ of 2,4-D $+0.5 \mathrm{mg} / \mathrm{l}$ of $\mathrm{Kin}$ is essential for the formation of MCS and ELS. Among the three genotypes studied, cv. Emir showed the highest androgenic efficiency.

One of the most vital parameters for androgenesis is the selection of the proper bud size and developmental stage of microspore for culture initiation (Constantin 1981; Ferrie and Caswell 2011). For legume species, some reports emphasize the correlation of bud size and microspore 


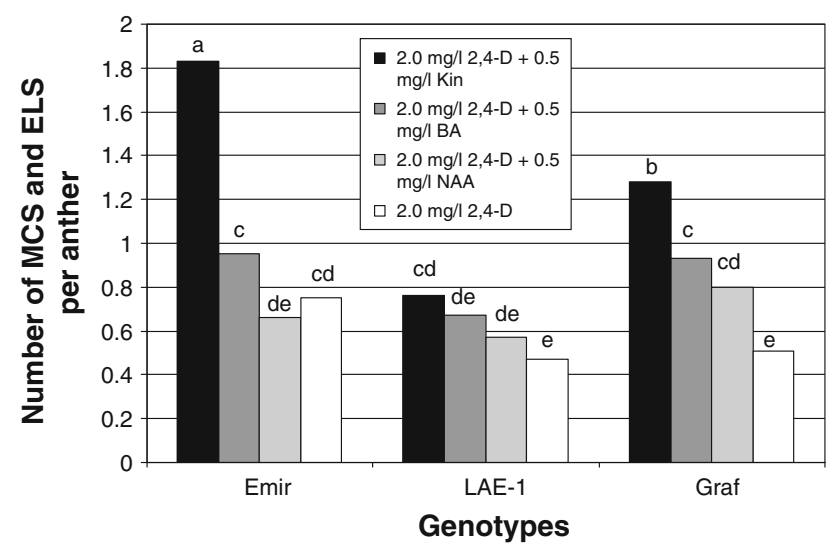

Fig. 5 Effect of interaction between genotypes and GRs on formation of multicellular and embryo-like structures for three genotypes of Lupinus angustifolius. Multicellular and embryo-like structures were counted at the end of induction period on day 28. $a, b, c$ mean values followed by the same letter are not significantly different at $P=0.05$ according to Duncan's test

developmental stage to the development of MCS and ELS. In chickpea, Croser et al. (2005) pointed out that bud size was an important determinant of embryo development. The authors stated that a bud size of 3-4 mm was most conducive to embryo development because these buds contained microspores at mid-late uninucleate stages. Studying three cultivars of soybean, Lauxen et al. (2003) showed that the optimal bud size for androgenesis was 2.5-3.5 mm for two cultivars. The authors stated that a bud size of 3-4 mm should be used for the third cultivar of soybean. To establish such correlation for lupin, we performed a comprehensive study for three genotypes of L. angustifolius. In the present study, we found that in all three genotypes, a bud size of 5-6 mm, located in the middle part of the inflorescences, showed the highest response towards induction. In the above mentioned bud size, the maximum number of microspores was at mid-late uninucleate stage and showed the highest response towards the formation of MCS and ELS. For lupin androgenesis, in the context of bud size, our results are in accordance with the study of Bayliss et al. (2004). These authors observed that a bud size of $5 \mathrm{~mm}$ containing the highest number of microspores at late-uninucleate stage were the most responsive to induction. Compared to Bayliss et al. (2004), in the present study, we have specified the localization of the buds with appropriate sizes within the inflorescence. In contrast to Lauxen et al. (2003), we observed no significant difference (maximum 5\%) among the genotypes and the developmental stages of microspores from the middle segments of the inflorescences.

For culture initiation and further development, a high percentage of viable pollen could be a significant parameter. We checked the viability of microspores from the anthers of three segments of inflorescences. Microspore viability was over $90 \%$ for all three genotypes of $L$. angustifolius. Cold pre-treatment of inflorescences did not have any effect on the viability of microspores. In the present report, we also show the difference in anther colour of the anthers depending on their location in the buds of different segments of inflorescences. The anthers obtained from segment II were yellowish green in colour compared to the anthers from segments I and III, which were green and bright yellow, respectively. Cytological study shows that yellowish green anthers contained the maximum number of microspores at mid-uninucleate stage and showed the highest response towards induction. The differentiation of anthers owing to their colours, and their subsequent behaviour in culture, can be useful for establishing the initial selection criterion for androgenesis of L. angustifolius.

In the current investigation, formation of multicellular and embryo-like structures was observed. There was a clear demarcation in the developmental pattern between the microspores from the anthers of untreated and treated inflorescences. In case of untreated inflorescences, microspores increased in size due to the accretion of granular structures, followed by symmetrical division of the vegetative nucleus. Division of the nucleus continued, eventually leading to the formation of multicellular structures. In case of cold pre-treated inflorescences, in comparison to untreated inflorescences, a remarkable increase in microspore size was observed. Due to the excessive granular accumulation, nuclei were not visible, and hence it was not possible determine which of the nuclei continued to divide. At the end of induction period, an oval shaped embryo-like structure was formed. Formation of such ELS is also reported by Zagorska et al. (1997) from the microspores in the anther culture of Medicago sativa. During their further development, these structures were visible as white embryo-like structures on the embryogenic calli and finally converted into plants with different ploidy level. In our study, callus formation started during the induction period and continued on regeneration medium. Further development of ELS was observed as white embryo-like structures during callus formation. However, on regeneration medium, callus formed roots and the ELS did not show any further development. At the end of 1 month on regeneration medium, the calli turned brown and died. One possible reason for limiting further development of these structures into plants could be the non-optimized regeneration medium. The other factor affecting the release of MCS and ELS could be the strong exine layer, which is difficult to rupture. In case of lupin, Bayliss et al. (2004) also observed such hindrance in the release of MCS. We suggest that a combination of cold pre-treatment with certain other treatments such as application of osmotic stress and/or suitable 
GR combinations can be helpful for release and further development of the observed MCS and ELS.

There have been few investigations on lupin androgenesis (Sator et al. 1983; Sator 1985; Ormerod and Caligari 1994; Bayliss et al. 2004; Skrzypek et al. 2008). Sator (1985) could regenerate plants from anther culture of L. polyphyllus; however, these plants were diploid. In rest of the reports, different conditions and media were tested for microspore and anther culture of lupin. Nevertheless, none of the approaches succeeded in overcoming the recalcitrance of lupins to androgenesis. Moreover, many differences were observed in the different studies. For instance, Ormerod and Caligari (1994) and Skrzypek et al. (2008) report the formation of MCS and ELS without any pre-treatment of buds or inflorescences. In contrast, our results support the study of Bayliss et al. (2004), who report the importance of cold pre-treatment for the development of MCS and ELS in L. angustifolius. Interestingly, in our study, cold-pretreated microspores followed a distinct developmental pathway into ELS, clearly distinguishable from MCS formed without cold-pretreatment. In all the above mentioned reports, no such case was observed.

We have analysed different media for anther culture of $L$. angustifolius. Our results show that for all three genotypes, the best response of microspores towards the formation of MCS and ELS was observed on MS medium supplemented with $2.0 \mathrm{mg} / \mathrm{l}$ 2,4-D + $0.5 \mathrm{mg} / \mathrm{l} \mathrm{Kin}$. The interaction genotype $\times$ GRs showed a statistically significant difference. Medium composition and growth hormones have a key role in the formation and further development of embryos (for review, see Jiménez 2005). The synthetic auxin 2,4-D has been reported to be an effective plant growth regulator for the induction of androgenesis in many plant species such as wheat (Ball et al. 1993) and legumes (Zhao et al. 1998; Croser et al. 2006). A concentration of $2.0 \mathrm{mg} / \mathrm{l}$ of 2,4-D along with different combinations of GRs was successfully used to achieve the induction of haploid embryos from anthers of soybean, followed by the regeneration of haploid plants (Zhao et al. 1998).

For androgenesis of Lupinus angustifolius, we have established the initial criteria for selection of anthers and microspores depending on their location in the buds. Our data show that for all three genotypes, a bud size of 5-6 mm (from the middle segment of inflorescence) contains anthers at the optimal developmental stage of microspores, necessary for androgenesis. Moreover, we show that anthers can be selected for culturing on the basis of their colour, depending on their location in the buds. We have obtained a significant number of MCS and ELS on MS medium supplemented with $2.0 \mathrm{mg} / 1$ 2,4-D + $0.5 \mathrm{mg} / 1$ Kin. These results will facilitate the selection of anthers and microspores of L. angustifolius for future research. Based on the obtained results, further studies are in progress for anther and isolated microspore culture of L. angustifolius.

Open Access This article is distributed under the terms of the Creative Commons Attribution Noncommercial License which permits any noncommercial use, distribution, and reproduction in any medium, provided the original author(s) and source are credited.

\section{References}

Ball ST, Zhou H, Konzak CF (1993) Influence of 2, 4-D, IAA and duration of callus induction in anther culture of spring wheat. Plant Sci 90:195-200

Bayliss KL, Wroth JM, Cowling WA (2004) Pro-embryos of Lupinus spp. produced from isolated microspore culture. Aust J Agr Res 55:589-593

Constantin MJ (1981) Pollen embryogenesis to induce, detect, and analyze mutants. Environ Health Persp 37:27-33

Croser J, Lülsdorf M, Davies P, Wilson J, Sidhu P, Grewal R, Allen K, Dament T, Warkentin T, Vandenberg A, Siddique K (2005) Haploid embryogenesis from chickpea \& field pea-progress towards a routine protocol. In: Proceedings of the Australian branch of the IAPTC\&B, 21-24th September, Perth, Western Australia, pp 71-82

Croser JS, Lülsdorf MM, Davies PA, Clarke HJ, Bayliss KL, Mallikarjuna N, Siddique KHM (2006) Toward doubled haploid production in the Fabaceae: progress, constraints, and opportunities. Crit Rev Plant Sci 25:139-157

Erdtman G (1952) Pollen morphology and plant taxonomy-Angiosperms (an introduction to palynology). Almqvist and Wiksell, Stockholm

Faluyi MA, Zhou XM, Zhang F, Leibovitch S, Migner P, Smith DL (2000) Seed quality of sweet white lupin (Lupinus albus) and management practice in eastern Canada. Eur J Agron 13:7-37

Ferrie AMR, Caswell KL (2011) Isolated microspore culture techniques and recent progress for haploid and doubled haploid plant production. Plant Cell Tissue Organ Cult 104:301-309

Grewal RK, Lulsdorf M, Croser J, Ochatt S, Vandenberg A, Warkentin TD (2009) Doubled-haploid production in chickpea (Cicer arietinum L.): role of stress treatments. Plant Cell Rep 28:1289-1299

Huyghe C (1997) White lupin (Lupinus albus L.). Field Crop Res $53: 147-160$

Jiménez VM (2005) Involvement of plant hormones and plant growth regulators on in vitro somatic embryogenesis. Plant Growth Regul 47:91-110

Kaur P, Bhalla JK (1998) Regeneration of haploid plants from microspore culture of pigeonpea (Cajanus cajan L.). Indian J Exp Biol 36:736-738

Lanas I, Gallego P, Martin L, Fernandez J, Alonsom A, Elena-Rosello J, Blazquez A, Villalobos N, Guerra H (2006) In vitro culture of Medicago arborea L. anthers: initial response. Plant Growth Regul 49:49-60

Lauxen MS, Kaltchuk-Santos E, Hu CY, Callegari-Jacques SM, Bodanese-Zanettini MH (2003) Association between floral bud size and developmental stage in soybean microspores. Braz Arch Biol Techn 46:515-520

Moraes AP, Bonadese-Zanettini MH, Callegari-Jacques SM, Kaltchuk-Santos E (2004) Effect of temperature shock on soybean microspore embryogenesis. Braz Arch Biol Techn 47:537-544 
Murashige T, Skoog F (1962) A revised medium for rapid growth and bio assays with tobacco tissue cultures. Physiol Plantarum 15:473-497

Ochatt S, Pech C, Grewal R, Conreux C, Lulsdorf M, Jacas L (2009) Abiotic stress enhances androgenesis from isolated microspores of some legume species (Fabaceae). J Plant Physiol 166: $1314-1328$

Ormerod AJ, Caligari PDS (1994) Anther and microspore culture of Lupinus albus in liquid culture medium. Plant Cell Tissue Organ Cult 36:227-236

Sator C (1985) Plant regeneration from anthers of lupins. Landbauforsch Volk 35:5-7

Sator C, Mix G, Menge U (1983) Investigation on anther culture of Lupinus polyphyllus. Plant Res Dev 18:37-46
Skrzypek E, Czyczylo-Mysza I, Marcińska I, Wędzony M (2008) Prospects of androgenetic induction in Lupinus spp. Plant Cell Tissue Organ Cult 94:131-137

Sudhersan C, Manuel SJ, Al-Sabah L (2008) Haploid plant production from pollen grains of Sturt's Desert Pea via Somatic Embryogenesis. American-Eurasian J Sci Res 3:44-47

Zagorska N, Dimitrov B, Gadeva P, Robeva P (1997) Regeneration and characterization of plants obtained from anther cultures in Medicago sativa L. In Vitro Cell Dev Biol Plant 33:107-110

Zhao G, Liu Y, Li J (1998) Germination of embryo in soybean anther culture. Chin Sci Bull 43:1991-1995 